\title{
The chemical composition of Amazonian plants $\left(^{*}\right)$
}

\section{A catalogue, edited by setor de fitoquímica, INPA, Manaus, Amazonas}

FAMILY

MYRISTICACEAE
SPECIE

Iryanthera elliptica Ducke

OCCURRENCE: Manaus, Amazonas

TrUNK WOOD:

\section{Sitosterol}

2-( $\omega$-piperonyltridecyl)-4-Methylidenetetronic acid (le-2)

Spiro-[4-methoxy-3,5-cyclohexadiene-1,1' (1',2',3, 4'-tetrahydro-6', 8'-dihydro-6', 8'-dihydroxy-7'-metho xynaphtalene]-2-one (le-3)

( \pm )-7,4'-Dihydroxy-3'-methoxyflavane (le-4)

1-(4'-hydroxy-2'-methoxyphenyl)-3-(4' '-hydroxy-3' '-methoxyphenyl)-Propane (le-5)<smiles>C=COC(=O)C(CCCCCCCCCCCCCc1ccc2c(c1)OCO2)=C(C)O</smiles><smiles>COC1=CC(=O)C2(C=C1)CCCc1cc(O)c(OC)c(O)c12</smiles>

$1 e-3$<smiles>CCc1ccc(O)cc1OC</smiles><smiles>COc1cc(C2CCc3ccc(O)cc3O2)ccc1O</smiles>

$1 e-4$<smiles>COc1cc(C)ccc1O</smiles>

$1 e-5$

\section{REFERENCES :}

1. Diaz Diaz, P. P. (1978) Taken from part of the D. Sc. Thesis submitted to Universidade de São Paulo, Brasil.

2. De Lima, R. A., Franca, N. C., Diaz Diaz, P. P., Gottlieb, O. R. (1975) Phytochemistry, 14,1831 .

$\left({ }^{*}\right)$ - Contributions to this catalogue, which will be continued in subsequent issues of this Journal, are invited and should be submitted to address give above. 
FAMILY

LEGUMINOSAE-LOTOIDAE

OCCuRRENCE: Manaus, Amazonas

TRUNK WOOD:

Elemicin

Biochanin-A

Naringenin (1)

Pratensein (2a)

5,7-Dihydroxy-8,3',4'-trimethoxyisoflavone (2b)

$5,7,3^{\prime}$-Trihydroxy-8,4'-dimethoxyisoflavone (2c)

$5,7,3^{\prime}\left(4^{\prime}\right)$-Trihydroxy-6,4' (3')-dimethoxyisoflavone (2d)<smiles>O=C1CC(c2ccc(O)cc2)Oc2cc(O)cc(O)c21</smiles>

(1)

$$
\begin{aligned}
& R=R_{1}=R_{5}=O M e \\
& R_{2}=R_{4}=O H \\
& R_{3}=H
\end{aligned}
$$

\section{REFERENCE :}<smiles>[R]c1ccc(-c2coc3c([R5])c([R6])c([R5])c([R2])c3c2=O)cc1[R]</smiles>

Albuquerque, Fabio Barbosa de (1978) Taken from part of the M. Sc. Thesis submitted to Univer. sidade Federal de Minas Gerais, Brasil. 